\title{
Thermal and hydrostatic structure of the protoplanetary nebula exposed to stellar radiation and stellar wind from the central star
}

\author{
Young Seok Yun ${ }^{1}$, Hiroyuki Emori ${ }^{2}$, and Kiyoshi Nakazawa ${ }^{1}$ \\ ${ }^{1}$ Department of Earth and Planetary Sciences, School of Science, Tokyo Institute of Technology, Tokyo 152-8551, Japan \\ ${ }^{2}$ Department of business management, Shumei University, Chiba 276-0003, Japan \\ (Received February 16, 2006; Revised October 26, 2006; Accepted December 9, 2006; Online published June 27, 2007)
}

\begin{abstract}
A model for a nebula exposed to the radiation and stellar wind from the central star has been examined. T Tauri stars commonly have disks and stellar wind, though we have no theoretical model on nebular thermal structure under the wind. The aims of this paper are to propose a theoretical nebula model, and to perform mathematical analysis on the geometrical structure and temperature distribution of a passive nebula. Its geometrical surface is determined by the dynamical pressure of the stellar wind. The nebular surface is assumed to be a black body surface, heated by the incident stellar photon flux. We obtain temperature from the equation of energy balance between the stellar radiation upon this nebular surface and the black body radiation from it. The temperature distribution in passive disks is insensitive to the wind strength and is almost identical even if the wind strength changes by five orders of magnitude. The nebula temperature is not expressed by a simple power law function of the distance from the central star. This is an important difference between our results and those of previous works. Since the nebula surrounding a T Tauri star is influenced by stellar wind, our model may be more appropriate than any other simple single power law temperature for passive protoplanetary nebulae.
\end{abstract}

Key words: Protoplanetary nebula, stellar wind, passive nebula.

\section{Introduction}

According to the standard model of planetary formation (Safronov, 1969; Hayashi et al., 1985), planets were formed in a disk-like nebula surrounding the Sun in the pre-mainsequence stage. Nebulae surrounding pre-main-sequence stars have been studied through astronomical observations of IR emission excess from T Tauri stars (e.g., Adams et al., 1987; Rydgren and Zak, 1987; Beckwith et al., 1990). From observational data, it has already become clear that nebulae have two different phases, namely, an active phase and a passive phase.

Nebulae in an active phase emit energy on their own and show characteristic features in their spectral energy distribution (Lynden-Bell and Pringle, 1974; Adams et al., 1987, 1988). In order to reproduce observational features, a number of researchers have studied disk accretion models (e.g., Lin and Papaloizou, 1985; Papaloizou and Lin, 1995) in which the emitted energy is supplied from the gravitational energy released by mass accretion toward the central star. These studies tell us that the mass accretion rates in active nebulae are in the range of $1 \times 10^{-8} M_{\odot} \mathrm{yr}^{-1}$ to $1 \times 10^{-5} M_{\odot} \mathrm{yr}^{-1}$ (e.g., Carr, 1989; Hartigan et al., 1995; Boss, 1996; Kenyon et al., 1996; Calvet, 1997). Such strong mass flow toward the central star continues for a few million years (e.g., Hartmann et al., 1998). Nebulae cease the emission and only reflect radiation from the central star. This means that disk nebula comes into a passive phase and

Copyright (c) The Society of Geomagnetism and Earth, Planetary and Space Sciences (SGEPSS); The Seismological Society of Japan; The Volcanological Society of Japan; The Geodetic Society of Japan; The Japanese Society for Planetary Sciences; TERRAPUB. settles in a hydrostatic equilibrium state. In such a passive nebula, the planetary formation process starts (Hayashi et al., 1985).

In the passive phase, the nebular temperature is essentially determined by the irradiation from the central $\mathrm{T}$ Tauri star. Kusaka et al. (1970, which is called K70 hereafter) first studied the temperature distribution of the passive nebula assuming the following situations :

1) The nebula is optically thick and hydrostatic;

2) The nebula is isothermal vertically;

3) The height of a nebular black body surface coincides with the scale height of the nebula;

4) The temperature is determined by the energy balance between irradiation from the central star and black body radiation from the nebular surface.

Another temperature model of the passive nebula disk was presented by Chiang and Goldreich (1997, called CG97 hereafter). Although CG97 considered the energy balance on a surface to be the same as K70, they took into account an optically thin, slightly high-temperature region on the top of the nebula in order to reproduce double peak features in SED (e.g., Rydgren and Zak, 1987; Beckwith et al., 1990). Furthermore, they supposed that exactly half of the radiation reprocessed by the surface layer escapes directly into space, with the remaining half being transfered towards the disk interior. After CG97, Chiang et al. (2001, called C01 hereafter) refines the model of CG97 improving the numerical procedure.

One of characteristics observed for pre-mainsequence stars is stellar wind. Most classical T Tauri stars have ex- 
tremely strong winds (Kuhi, 1964; Carr, 1989; Storm et al., 1989; Greene and Mayer, 1995; McCaughrean and O’Dell, 1996). This strong wind is closely related with disk accretion. As a protoplanetary nebula evolves from an active phase to a passive phase, the stellar wind from the central star may change from a strong accretion-driven wind to a weak spherically wind, which resembles the stellar wind from the sun. Unfortunately, we have few observations of stellar wind from solar-like stars (Wood et al., 2002).

The observations for the stellar wind from solar-like stars tell us that the mass loss rates among them distribute in the range between almost equal to the rate of the present solar wind and about thirty fold larger than it. It is accepted that the origin of these wind is surface activities of the central star; for example sun's hot corona. Because these surface activities may exist on the surface of the T Tauri stars, we believe that there is some kind of stellar wind flow from the central stars which have the passive protoplanetary nebula. However, we can not find any observational information of the stellar wind from $\mathrm{T}$ Tauri stars with passive phase disk.

The stellar wind from the central star with passive nebula is different from the wind of active accretion disk. It may be a wind-like present solar wind. So the mass loss rate of the wind from a star with a passive disk is almost equal to or greater than that of wind from solar-like stars, because $\mathrm{T}$ Tauri stars even in the weakline stage present stronger surface activities; for example, line emissions, than stars in the mainsequence stage (e.g., Walter et al., 1988; Basri and Bertout, 1989; Bertout, 1989). Based on the stellar wind from solar-like stars (e.g., Hartmann, 1985; Woods et al., 2002), it is plausible that the mass loss rates for the $\mathrm{T}$ Tauri star with a passive disk is in the range of the larger than present solar wind by about two or three orders of magnitude. If the nebula is exposed to the stellar wind, what kind of effect is brought to the nebula? In this paper we cencentrate on this question. The mass loss rates of the wind, however, remain unclear owing to the lack of observations.

Owing to this uncertainty in mass loss rates, we consider that it is more important now to make clear the physicalin some cases mathamatical-behavior of the solution expressing temperature distribution of a passive nebula rather than to specify a model under suspicious estimates on the mass loss rates. So we change the mass loss rates in a range that seems nonsense if we adopt it as a rate of T Tauri star without any active accretion disk. It should be noted that the mass loss rates chosen in this study are not adequate for real $\mathrm{T}$ Tauri stars with a passive disk. It is very important to enlarge the range of mass loss rate because an important behavior of basic equations was found in this wide range parameter study.

Hereafter we will reconstruct the passive nebula model exposed to both of the stellar radiation and the stella wind. Specifically, the aims of our this paper are

1) to develop basic equations which describe the thermal and hydrostatic structure of the passive nebula exposed to the stellar wind and stellar radiation;

2) to determine a characteristic behavior of solutions described by the basic equations;
3) to investigate the geometrical structure and the temperature distribution of the passive nebula exposed to the wind.

In Section 2, we will describe the assumptions adopted in this paper (including the stellar wind model and the nebula model) as well as the basic equations that describe the thermal structure and the geometrical figure of the nebula. We will then present the characteristic behavior of solutions for the basic equations in Section 3. On the basis of the results presented Section 3, we will present in Section 4 the thermal structure and geometry of the nebula surface exposed to stellar wind with various strengths. We will see that the temperature distribution and the geometrical figure of the nebula depend very weakly on the strength of the stellar wind as long as the wind strength is weaker than critical strength. In Section 5, the validity of the adopted assumptions and simplifications will be checked with positive results. Finally, conclusions and discussion will be described in Section 6.

\section{Basic Equations}

We suppose that a protoplanetary nebula, surrounding a $\mathrm{T}$ Tauri star, is exposed to the stellar wind from the central star. The nebula is in a passive phase, namely, it has no energy source within it. The temperature of the nebula is determined essentially by the irradiation of photons from the central star. In order to see the geometric figure and the temperature distribution of the nebula, we make, for simplicity, the following assumptions on the passive nebula and the stellar wind:

1) The nebula is axi-symmetric and mirror-symmetric with respect to the midplane of the nebula (hereafter, we use cylindrical coordinates $(r, z)$ the origin of which is at the center of the central star (see Fig. 1)). Furthermore, the nebula is geometrically very thin.

2) The nebula is in a hydrostatic equilibrium state and isothermal in the z-direction (i.e., in the direction perpendicular to the midplane of the nebula).

3) The nebula is composed of an ideal gas with the mean molecular weight, $\mu(=2.34)$.

4) The radial distribution of the nebular mass is described by a power law of $r$ before a part of it is blown off by the stellar wind, i.e.,

$$
\Sigma=\Sigma_{0}\left(\frac{r}{1 \mathrm{AU}}\right)^{\gamma}
$$

where $\Sigma$ is the surface density of the nebula and $\Sigma_{0}$ is that at $r=1 \mathrm{AU}$. We consider a protoplanetary nebula after the minimum-mass solar nebula model (Hayashi, $1981)$, in which $\Sigma_{0}$ and $\gamma$ are equal to $1.7 \times 10^{3} \mathrm{~g} / \mathrm{cm}^{2}$ and $-3 / 2$, respectively.

5) The nebula is optically thick (i.e., the nebula catches the photon irradiation from the central star just at the surface) and has no heat source inside it.

6) The wind is steady and spherically symmetric and the wind velocity is constant both spatially.

7) On the contact surface between the nebula and the stellar wind, the dynamical pressure of the wind balances with hydrostatic pressure in the nebula. 


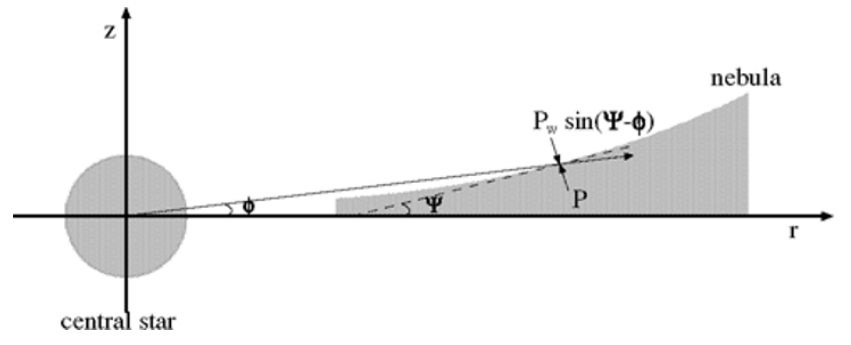

Fig. 1. Schematic figure of the contact surface between the stellar wind and the nebula gas. Two angles $\phi$ and $\Psi$ are $\tan ^{-1}\left(z_{s} / r\right)$ and $\tan ^{-1}\left(d z_{s} / d r\right)$, respectively, where $z_{s}$ is the nebular height. $P$ and $P_{\mathrm{w}}$ are the hydrostatic pressure in the nebula and the dynamical pressure of the wind, respectively.

From assumptions (1), (2), and (3), we obtain the hydrostatic pressure of the nebula at height $z$ as

$$
P=\frac{k T}{\mu m_{\mathrm{H}}} \frac{\Sigma}{\sqrt{2 \pi} H} \exp \left(-\frac{z^{2}}{2 H^{2}}\right)
$$

(e.g., Hayashi et al., 1985), where $T$ is the nebula temperature at the distance $r, k$ is the Boltzmann constant, and $m_{\mathrm{H}}$ is the mass of hydrogen atom. Furthermore, $H$ is the pressure scale height of the nebula given by

$$
H=r\left(\frac{k T r}{\mu m_{\mathrm{H}} G M_{*}}\right)^{\frac{1}{2}},
$$

where $G$ is gravitational constant, and $M_{*}$ is the mass of the central star.

According to assumption (6), physical properties of the stellar wind are characterized completely by two parameters, the wind velocity, $v_{\mathrm{w}}$, and the mass loss rate, $\dot{M}_{*}$. For later use, we write these in the following forms:

$$
\begin{gathered}
v_{\mathrm{w}}=\beta\left(\frac{2 \mathrm{G} M_{*}}{R_{*}}\right)^{\frac{1}{2}} \\
\dot{M}_{*}=\frac{M_{*}}{t_{*}}
\end{gathered}
$$

In the former equation, $\beta$ is ratio of the wind velocity to the escape velocity, $R_{*}$ is the radius of the central star, and $t_{*}$ is a typical mass loss time of the central star. By the use of $\dot{M}_{*}$ and $v_{\mathrm{w}}$, the mass density of the wind, $\rho_{\mathrm{w}}$, is readily described by

$$
\rho_{\mathrm{w}}=\frac{\dot{M}_{*}}{4 \pi r^{2} v_{\mathrm{w}}} .
$$

Furthermore, the dynamical pressure of the wind, $P_{\mathrm{w}}$, is given by

$$
P_{\mathrm{w}}=\frac{M_{*}}{4 \pi r^{2}} \frac{\beta}{t_{*}}\left(\frac{2 \mathrm{G} M_{*}}{R_{*}}\right)^{\frac{1}{2}} .
$$

Note that the dynamical pressure is determined by only one parameter $t_{*} / \beta$. It is generally accepted that the stellar wind has a velocity comparable to or slightly larger than the escape velocity from the stellar surface (Parker, 1960, 1964). So it may safely be said that $\beta$ is almost equal to unity.

As mentioned, the mass loss rate of $\mathrm{T}$ Tauri stars with a passive disk is still unknown. In this study we choose an extreme wide range for the parameter $t_{*}$ as the values of $t_{*} / \beta, 1 \times 10^{8}, 1 \times 10^{9}, 1 \times 10^{10} \mathrm{yrs}$, and $1 \times 10^{12} \mathrm{yrs}$.

We now consider the geometry of a contact surface (which hereafter is called simply a surface) between the protoplanetary nebula and the wind. We show an edge-onview of the nebula in Fig. 1, in which two angles $\Psi$ and $\phi$ are introduced to describe the geometry of the surface. The angle $\Psi$ is between the midplane and the tangential line of the surface and $\phi$ is defined as $\tan ^{-1}\left(z_{s} / r\right)$ where $z_{s}$ is a height of the surface. Using this two angles we introduce a new variable, $f$, defined by

$$
f=\sin (\Psi-\phi) .
$$

As seen from Fig. 1, $f$ represents the $r$-component of a unit vector perpendicular to the surface of the nebula. In this study, $f$ is called a flaring index hereafter. Note that we can impose a geometrical relation between $z_{s}$ and $f$, i.e.,

$$
\frac{d z_{s}}{d r}=\frac{r f+z_{s} \sqrt{1-f^{2}}}{r \sqrt{1-f^{2}}-z_{s} f} .
$$

On the surface of the protoplanetary nebula, we have two equations describing dynamical and thermal balances. The wind dynamical pressure on the surface must balance with the hydrostatic pressure, $P$, of the nebula. Taking into account that the surface and velocity of wind takes an angle $(\Psi-\phi)$, we formulate the equation as

$$
P=f P_{\mathrm{w}} \quad \text { at } \quad z=z_{s},
$$

to express this dynamical balance, where $P$ and $P_{\mathrm{w}}$ are given by Eqs. (2) and (7), respectively. On the other hand, the nebular temperature is determined by a balance of radiative equilibrium. With the help of assumption (5), a radiative equilibrium condition is given by

$$
\begin{aligned}
\sigma T^{4}= & \frac{\sigma T_{*}^{4}}{\pi} \frac{R_{*}^{2}}{r^{2}}\left\{\frac{\pi}{2} f \frac{1+z_{s} / r}{\sqrt{1+\left(z_{s} / r\right)^{2}}}\right. \\
& +\frac{2 R_{*}}{3 r}\left[8 f \frac{z_{s} / r}{1+\left(z_{s} / r\right)^{2}}\right. \\
& \left.\left.+\frac{1-d z_{s}^{2} / d r^{2}}{\sqrt{1+\left(d z_{s} / d r\right)^{2}} \sqrt{1+\left(z_{s} / r\right)^{2}}}\right]\right\},
\end{aligned}
$$

where $T_{*}$ is the temperature of the stellar photosphere and $\sigma$ is the Stefan-Boltzmann constant (see K70). On the right-hand side of this equation, the first term in the large parenthesis represents the heating due to disk flaring and the second term represents the heating from the finite size of the central star. The assumption that the contact surface of the nebula is black body surface for absorption of radiation from central star will be confirmed in Section 5.

As seen later, the flaring index, $f$, is very small compared to unity and also $z_{s} / r \ll 1$ after assumption (1). So, we can neglect terms of $\left(z_{s} / r\right)^{2},\left(d z_{\mathrm{s}} / d r\right)^{2}, d z_{\mathrm{s}}^{2} / d r^{2}, f^{2}$, and $f\left(z_{s} / r\right)$ in Eqs. (9) to (11). Furthermore, to simplify the expression of the equations, we introduce dimensionless variables, $\eta, v, u$, non-dimensional numbers $M$, and $N$ :

$\eta=\left(\frac{r}{R_{*}}\right), v=\left(\frac{z_{s}}{r}\right), u=\left(\frac{T}{T_{*}}\right)\left(2 \eta^{2}\right)^{\frac{1}{4}}, N=2^{\frac{1}{4}}\left(\frac{T_{\mathrm{g}}}{T_{*}}\right)$, 


$$
M=2^{\frac{7}{8}} \sqrt{\pi} \mathrm{G} \Sigma_{0} \sqrt{\frac{R_{*}}{2 \mathrm{G} M_{*}}} \frac{t_{*}}{\beta}\left(\frac{T_{*}}{T_{\mathrm{g}}}\right)^{\frac{1}{2}}\left(\frac{R_{*}}{1 \mathrm{AU}}\right)^{\gamma}
$$

In the above, $T_{\mathrm{g}}$ is the temperature corresponding to the gravitational potential energy at the stellar surface, given by (CG97)

$$
T_{\mathrm{g}}=\frac{\mathrm{G} M_{*} \mu m_{\mathrm{H}}}{2 k R_{*}} .
$$

Using these dimensionless variables and neglecting small quantities mentioned above, we can rewrite Eqs. (9) to (11) into the following simple forms:

$$
\begin{gathered}
\frac{d v}{d \eta}=\frac{f}{\eta} \\
f=M \eta^{\gamma+\frac{1}{4}} \sqrt{u} \exp \left(-N \frac{v^{2}}{\sqrt{\eta} u}\right) \\
u^{4}=f+\frac{4}{3 \pi} \frac{1}{\eta}
\end{gathered}
$$

Combining Eqs. (14), (15), and (16), we obtain an equation for the flaring index, $f$, which is given by

$$
\mathcal{L} \frac{d \ln f}{d \ln \eta}=\mathcal{R},
$$

where

$$
\begin{gathered}
\mathcal{L}(f, \eta)=1-\frac{f}{8 u^{4}}(1+2 s), \\
\mathcal{R}(f, \eta)=\left(\gamma+\frac{1}{4}\right)-\frac{1}{6 \pi} \frac{1+2 s}{\eta u^{4}}+\frac{s}{2}\left(1-4 \frac{f}{v}\right),
\end{gathered}
$$

and

$$
s \equiv N \frac{v^{2}}{\sqrt{\eta} u} .
$$

Equation (17) is the basic equation to be solved. In our present study, supposing a nebula around typical $\mathrm{T}$ Tauri stars we choose parameters on the central star as $T_{*}=4000$ $\mathrm{K}, M_{*}=1 M_{\odot}$, and $R_{*}=2 R_{\odot}$ (e.g., Beckwith et al., 1990).

\section{Choice of a Solution}

Prior to the description of numerical results, we will discuss in short the behavior of solutions to Eq. (17). As seen later, such a discussion gives us useful insight on demanding conditions to obtain proper solutions for the nebula models.

Usually we have to impose an appropriate boundary condition on $f$ at the outer (or inner) boundary of the nebula in order to solve Eq. (17). However, it is difficult to determine the position of the outer and/or inner boundaries of the nebula, because the nebula may extend in a radial direction continuously to the ambient molecular cloud surrounding the protoplanetary nebula. In this study, we determine, by an artificial manner, the inner boundary at $\eta=3$ (=0.03 AU), which corresponds to three fold larger as the radius of the central star, i.e., $r=3 R_{*}$. As will be seen later, the temperature at $\eta=3$ is equal to $1200 \mathrm{~K}$, independent of the choice of the wind parameter $t_{*} / \beta$ and to the value of $f$. This temperature is almost equal to the vaporization

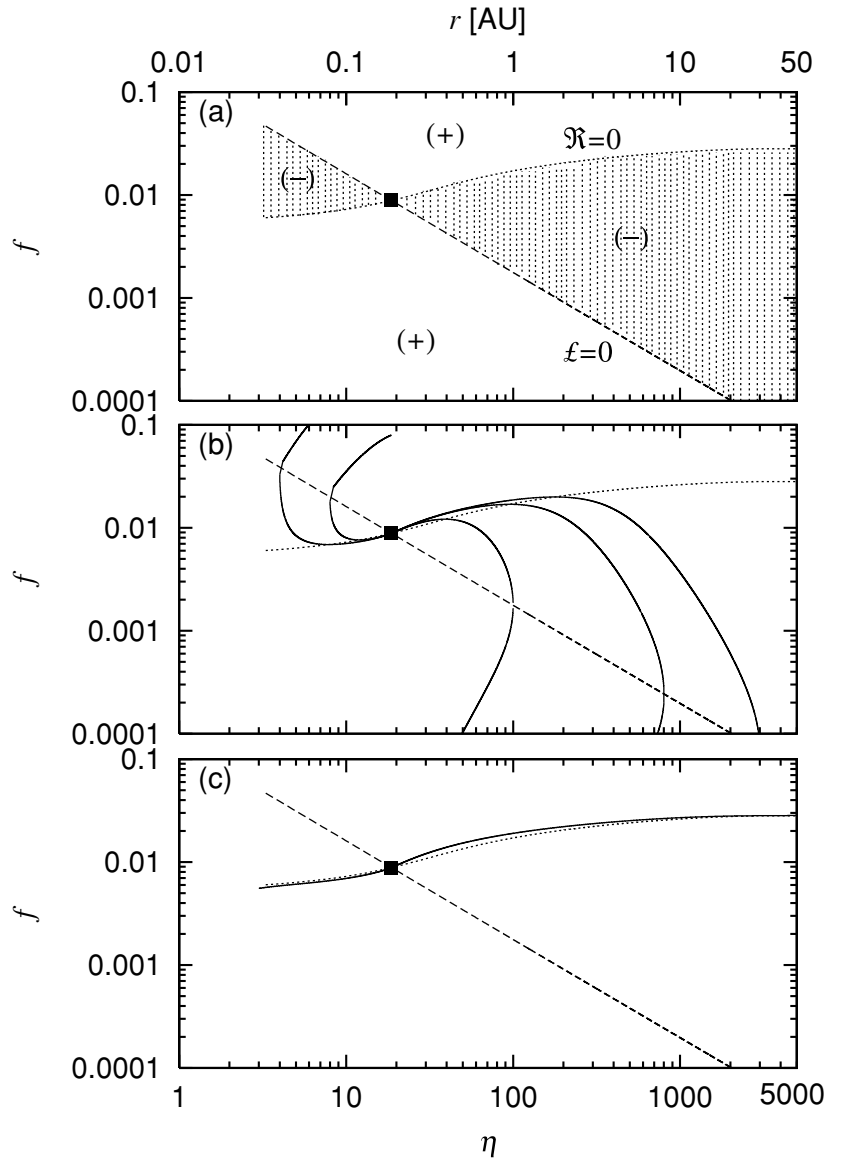

Fig. 2. (a) The null- $\mathcal{L}$ (dashed line) and the null- $\mathcal{R}$ (dotted line) lines for the case of $t_{*} / \beta=1 \times 10^{8} \mathrm{yrs}$. The critical point, in which both $\mathcal{L}$ and $\mathcal{R}$ vanish simultaneously, is shown by black square. The two curves divide the $\log f-\log \eta$ plane into four regions; In regions denoted by $(+)$ (or $(-)$ ), solution curves have positive (or negative) gradients. (b) Solution curves which cross the null- $\mathcal{L}$ curve at points other than the critical points. (c) Solution curves which pass through the critical point with a certain appropriate gradient, $(d \ln f / d \ln \eta)_{\mathrm{c}}$.

temperature of typical silicates. So, no dust grain can exist in a region inside this boundary. This is the reason why we choose the inner boundary as mentioned above. As for the outer boundary, we choose $\eta=5 \times 10^{3}$ (i.e., $r=50 \mathrm{AU}$ ). Although we determine the inner and outer boundaries, we cannot find physical conditions at the boundaries. So, we have to overcome this difficulty from a completely different point of view.

It should be noted that there are two characteristic curves corresponding to $\mathcal{L}=0$ and to $\mathcal{R}=0$ on $\log \eta$-log $f$ plane (see Eq. (17)) and that there is a critical point on which both $\mathcal{L}$ and $\mathcal{R}$ vanish simultaneously (hereafter, $\eta$ and $f$ at the critical point are denoted by $\eta_{\mathrm{c}}$ and $f_{\mathrm{c}}$, respectively).

They are plotted in Fig. 2(a), as an example, for the case of $t_{*} / \beta=1 \times 10^{8} \mathrm{yrs}$. On the lines of $\mathcal{R}=0$ and $\mathcal{L}=0$, solution curves run horizontally and vertically, respectively, in the $\log \eta-\log f$ plane. The $\log \eta-\log f$ plane is divided into four regions by the two curves. In two regions denoted by (-) in Fig. 2(a), solution curves have a negative gradient, i.e., $d \ln f / d \ln \eta<0$, whereas in two regions denoted by $(+), d \ln f / d \ln \eta$ becomes positive. We can readily see from Fig. 2(b) that if a solution crosses the null- $\mathcal{L}$ curve at 


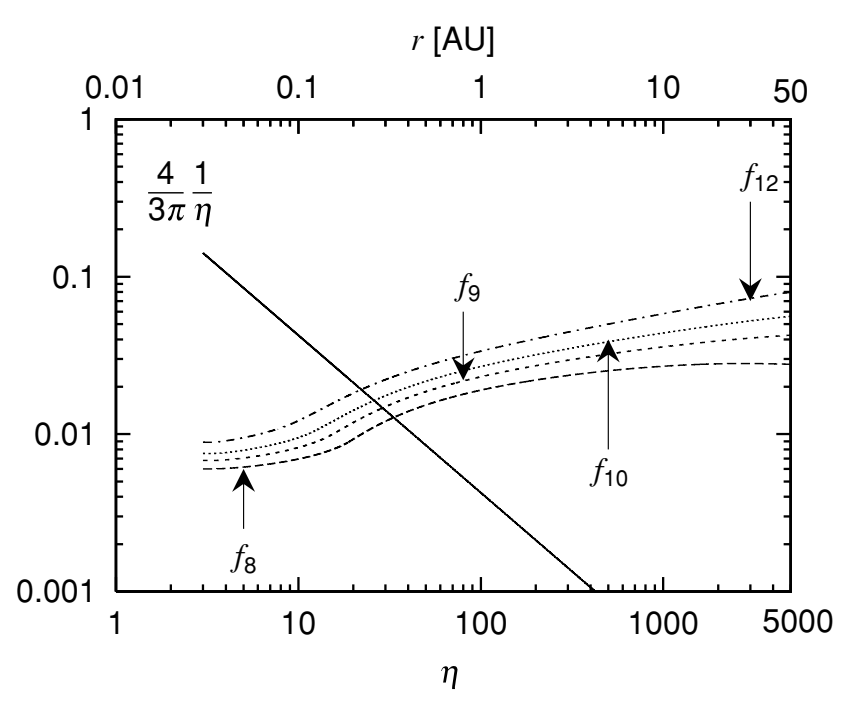

Fig. 3. Flaring index $f$ as a function of $\eta$ for the four different values of $t_{*} / \beta$. In the figure, $f_{\mathrm{n}}$ denotes the result for the case of $t_{*} / \beta=1 \times 10^{\mathrm{n}} \mathrm{yrs}$. For the later use, we also plot the line $4 / 3 \pi \eta$ which is the second term of Eq. (16).

$\eta$ larger (or smaller) than $\eta_{\mathrm{c}}$, it cannot extend to an outer (or inner) direction any further. On the other hand, as discussed in detail in Appendix A, only when solutions pass through the critical point with certain appropriate gradients $(d \ln f / d \ln \eta)_{\mathrm{c}}$ is there a possibility that we have solutions which describe models of the nebula extending infinitely.

Taking account of the above discussion about the behavior of solutions, we demand, a priori, the following two conditions to choose an appropriate solution. That is,

1) A solution curve should pass through the critical point continuously;

2) Flaring index $f$ should be always an increasing function of $\eta$ in the whole region between $0.03 \mathrm{AU}$ and $50 \mathrm{AU}$.

Even if we impose the conditions, we cannot specify a solution because many solutions satisfy the above two conditions. So, for the practical treatment, we impose the condition, $\mathcal{R}=0$, on the outer boundary, which is determined rather artificially at $50 \mathrm{AU}$ from the central star as illustrated in Fig. 2(c). We discuss in detail our choice of the boundary conditions in Appendix B.

\section{Results}

By solving Eq. (17) numerically, the nebular models are obtained for four cases of the wind parameter, $t_{*} / \beta=$ $1 \times 10^{8}, 1 \times 10^{9}, 1 \times 10^{10}$, and $1 \times 10^{12} \mathrm{yrs}$. The flaring indices, $f$, of the nebula are plotted in Fig. 3 for the four cases. From this figure we can readily see the following three results. One is that $f$ does not depend so strongly on the wind parameter $t_{*} / \beta$; the difference in $f$ is confined within a level of only a factor of 5 or less although the wind parameter changes over four orders of magnitude, i.e., from $1 \times 10^{8} \mathrm{yrs}$ to $1 \times 10^{12} \mathrm{yrs}$. Secondly, the flaring index becomes large when the wind parameter is large. This is a natural result because the wind pressure, which suppresses the nebular gas, increases with a decrease in

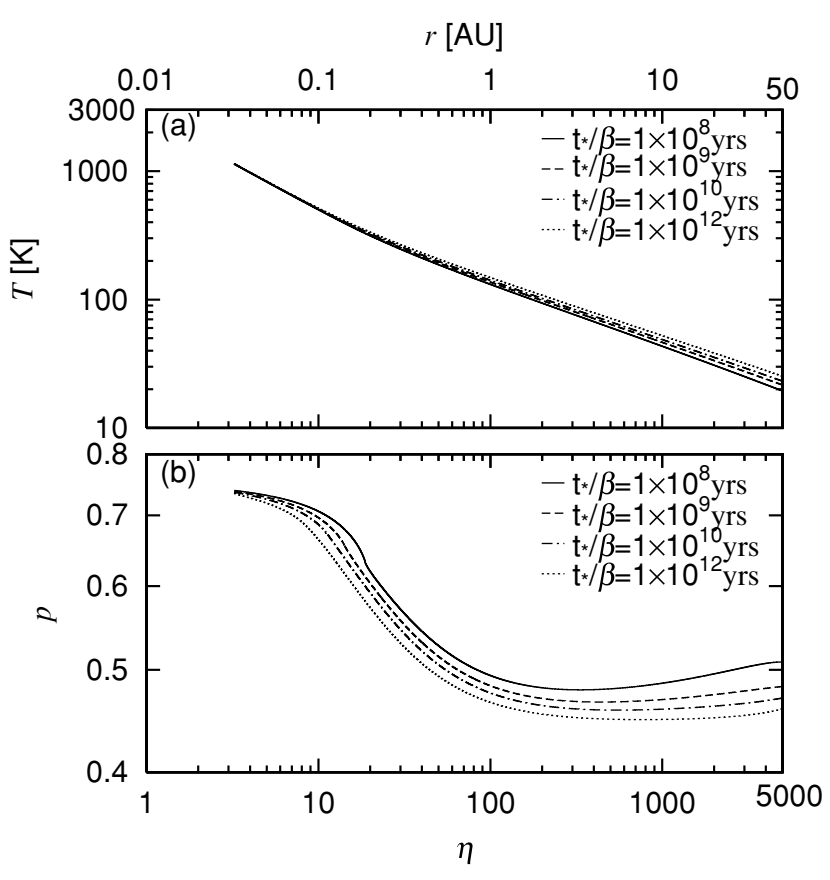

Fig. 4. Temperature distributions of the nebula for the four cases of $t_{*} / \beta$ from $1 \times 10^{8}$ to $1 \times 10^{12}$ yrs (Panel (a)). We also show the corresponding power-law index $p$ defined as $p=-d \ln T / d \ln r$ in Panel (b).

$t_{*} / \beta$ (i.e., with an increase in the wind strength). Finally, owing to the suppression by the wind pressure, the flaring index is confined within a very low level throughout the region even in the case of the weak wind i.e., the case of $t_{*} / \beta=1 \times 10^{12} \mathrm{yrs}$.

Corresponding temperature distributions of the nebula are plotted in Fig. 4(a) for the four cases of the wind parameter $t_{*} / \beta$. In the inner region of the nebula, where $r \leq 0.1 \mathrm{AU}$, the temperatures of the nebula for the four different wind parameters coincide with each other perfectly. This is due to the fact that the temperature in this region is determined by radiation from the finite size of the central star since the flaring index $f$ is very small compared to $4 / 3 \pi \eta$ (see Fig. 3 as well as Eq. (16)). In the outer region ( $r \geq 1 \mathrm{AU})$, on the other hand, the temperature distribution depends slightly on the wind parameter $t_{*} / \beta$. In this region, the temperature is determined by the flaring index $f$; in other words, the first term on the right hand side of Eq. (16) is larger than the second term (see Fig. 3).

The temperature of the nebula behaves in the same manner as the flaring index $f$, because it is nearly proportional to $f^{1 / 4}$. So, the temperature becomes lower when the stellar wind is stronger as long as we compare the temperatures at the same position in the nebula.

In order to study the temperature distribution of the nebula in detail, we plot the power-law index $p$ in Fig. 4(b), which is defined as

$$
p=-\frac{d \ln T}{d \ln r}
$$

In the inner region where $r \leq 0.1 \mathrm{AU}, p$ is confined within a narrow range between 0.75 and 0.65 . This is readily understood in the following manner. Noting that, as mentioned before, $f$ is very small compared to $4 / 3 \pi \eta$ (see Fig. 3 ) and 


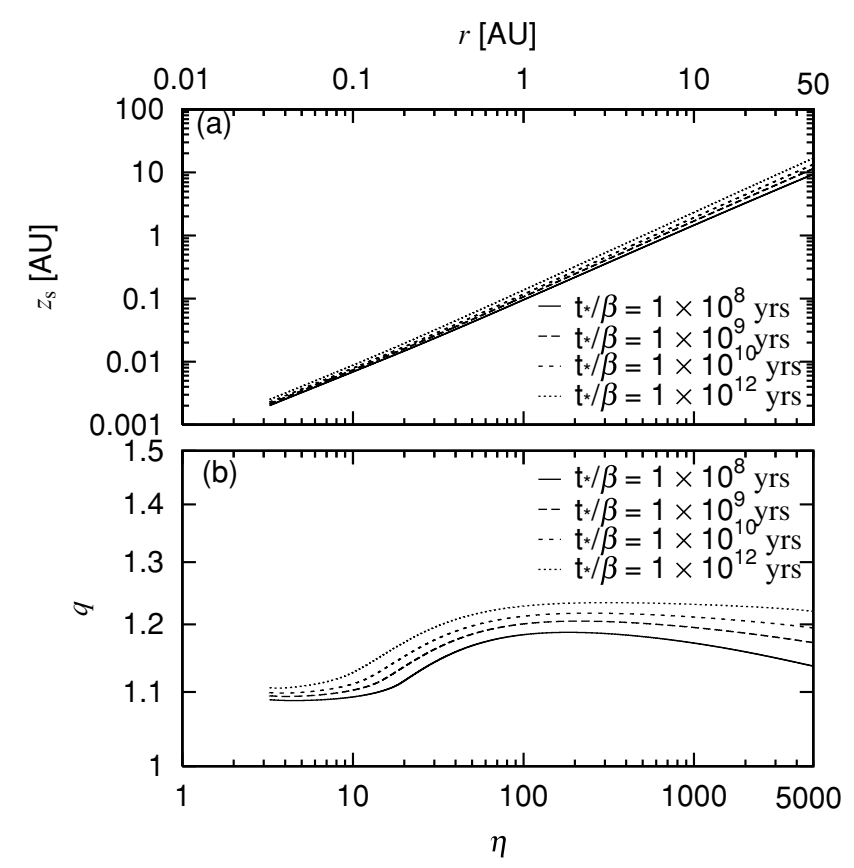

Fig. 5. The nebular heights $z_{\mathrm{S}}$ for the four cases of $t_{*} / \beta$ (Panel (a)) and the power-law index $q$, i.e., $d \ln z / d \ln r(\operatorname{Panel}(\mathrm{b}))$.

rewriting Eq. (16), we have an approximate formula

$$
p \simeq \frac{3}{4}-\frac{3 \pi}{16} f \eta\left(1+\frac{d \ln f}{d \ln \eta}\right) .
$$

Because the second term on the right hand side of the above equation is smaller than 0.1 (see Fig. 3), we have $p$ in the range mentioned above. On other hand, in the outer region ( $r \geq 1 \mathrm{AU}), p$ is almost constant but depends slightly on the adopted wind parameter $t_{*} / \beta$. Since $\eta f \gg 1$ in this region, $u$ is expressed as $f^{1 / 4}$ from Eq. (16). So the power index $p$ is given as

$$
p \simeq \frac{1}{2}-\frac{1}{4} \frac{d \ln f}{d \ln \eta}
$$

According to Fig. 3, $d \ln f / d \ln \eta$ increases slightly with an increase in $t_{*} / \beta$ and, hence, $p$ is smaller in the case of $t_{*} / \beta=1 \times 10^{12} \mathrm{yrs}$ than that in the case of $1 \times 10^{8} \mathrm{yrs}$. In the intermediate region between $0.1 \mathrm{AU}$ and $1 \mathrm{AU}$ the two terms on the right hand side of Eq. (16) switch the dominant role from the $4 / 3 \pi \eta$ term to the $f$ term. The power-law index $p$ changes greatly owing to this transition of heating sources in this region.

Using the results above, we can express the nebular temperature in an analytical but approximate manner. In the innermost ( $r \leq 0.1 \mathrm{AU})$ and the outer regions $(r \geq 1 \mathrm{AU})$, the power-law index, $p$, is, as a first approximation, $3 / 4$ and $1 / 2$, respectively. So, we have for the nebular temperature as a power-law function of the radial distance $r$

$$
T(r)=455\left(\frac{r}{0.1 \mathrm{AU}}\right)^{-3 / 4} \mathrm{~K} \quad \text { for } \quad r \leq 0.1 \mathrm{AU}
$$

and

$$
T(r)=145\left(\frac{r}{1 \mathrm{AU}}\right)^{-1 / 2} \mathrm{~K} \quad \text { for } \quad r \geq 1 \mathrm{AU} .
$$

Table 1. The mean power-law index $\bar{q}$ and the nebular height coefficient $z_{0}$.

\begin{tabular}{ccccc}
\hline$t_{*} / \beta(\mathrm{yrs})$ & $1 \times 10^{8}$ & $1 \times 10^{9}$ & $1 \times 10^{10}$ & $1 \times 10^{12}$ \\
$\bar{q}$ & 1.19 & 1.21 & 1.22 & 1.23 \\
$z_{0}(\mathrm{AU})$ & 0.11 & 0.12 & 0.13 & 0.15 \\
\hline
\end{tabular}

These expressions can be shown in another way: noting that $u^{4}=4 / 3 \pi \eta$ for $r \leq 0.1 \mathrm{AU}$ and using Eq. (12) we find $T=T_{*}\left(2 / 3 \pi \eta^{3}\right)^{1 / 4}$, i.e.,

$$
T(r)=0.68 T_{*}\left(\frac{r}{R_{*}}\right)^{-3 / 4} \quad \text { for } \quad r<10 R_{*} .
$$

Similarly, in the outer region where $u^{4}=f \simeq 0.04$ (see Fig. 3) we have for $T$

$$
T(r)=0.38 T_{*}\left(\frac{r}{R_{*}}\right)^{-1 / 2} \quad \text { for } \quad r>100 R_{*} .
$$

The heights of the nebula surface are shown in Fig. 5 for the four cases of $t_{*} / \beta$ from $1 \times 10^{8}$ yrs to $1 \times 10^{12} \mathrm{yrs}$. As seen from Fig. 5(a) they depend very weakly on the wind parameter $t_{*} / \beta$. As the wind parameter $t_{*} / \beta$ becomes larger, the height of the nebula becomes slightly larger. The reason is the same as that for the flaring index and the nebular temperature (see Figs. 3 and 4). In Fig. 5(b), we show the plot of the power index for the nebular height, $q$, defined as

$$
q=d \ln z_{\mathrm{s}} / d \ln r .
$$

Although the power-law index $q$ varies with the radial distance $r$, the change is not so large and is always confined within the range 1.1 and 1.3. As in the case of $z_{\mathrm{s}}$ itself, $q$ becomes small when the wind is strong because the flaring is suppressed by the strong wind pressure in this case. As long as we consider the nebula in the region $r \geq 1 \mathrm{AU}, q$ does not depend appreciably on the radial distance. Thus, the nebular height $z_{\mathrm{S}}(r)$ is approximated by power-law function:

$$
z_{\mathrm{s}}(r)=z_{0}\left(\frac{r}{1 \mathrm{AU}}\right)^{\bar{q}} \quad \text { for } \quad r \geq 1 \mathrm{AU} .
$$

The mean power law index $\bar{q}$ and the nebular height coefficient $z_{0}$, which are evaluated by our numerical simulations, are shown in Table 1.

Let us compare the nebular temperature obtained in the present study with those of the previous works by K70, CG97, and C01, in which they did not consider the effect of the stellar wind at all. To compare precisely, the quantities specifying the central star are set to be equal to those of CG97, that is, $M_{*}=0.5 M_{\odot}, R_{*}=2.5 R_{\odot}$, and $T_{*}=4000$ $\mathrm{K}$. In our model, $t_{*} / \beta$ is put to be $1 \times 10^{8} \mathrm{yrs}$. As mentioned before, the nebular height, $z_{\mathrm{s}}$, is approximated well by the power-law function of the radial distance in the region $r \geq$ $1 \mathrm{AU}$. The expressions predicted by our present model and other models are given by

$$
z_{\mathrm{s}}=\left\{\begin{array}{lll}
0.11\left(\frac{r}{1 \mathrm{AU}}\right)^{1.19} & \mathrm{AU} & \text { for ours } \\
0.17\left(\frac{r}{1 \mathrm{AU}}\right)^{9 / 7} & \mathrm{AU} & \text { for CG97 } \\
0.04\left(\frac{r}{1 \mathrm{AU}}\right)^{9 / 7} & \mathrm{AU} & \text { for K70 }
\end{array}\right.
$$




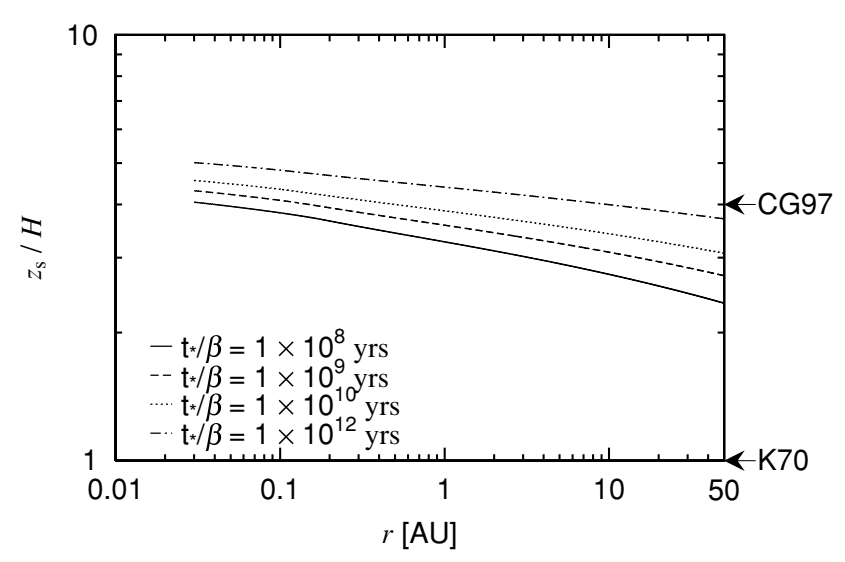

Fig. 6. The nebular heights $z_{\mathrm{s}}$ normalized by the scale height, $H$. In the models of K70 and CG97, $z_{\mathrm{s}} / H$ is fitted to be constant, namely 1 and 4 , respectively.

where we show our result for the case of $t_{*} / \beta=1 \times 10^{8} \mathrm{yrs}$ as an example. The difference in Eq. (30) becomes more clear by comparing $z_{\mathrm{s}} / H$ among the models where $H$ is the scale height given by Eq. (3). As seen from Fig. 6, the ratio between $z_{\mathrm{s}}$ and the scale height $H$ changes from 2.5 to 5 in the nebula, contrary to CG97 and K70. They assumed that the nebular surface is always equal to or five fold taller than $H$.

The nebula temperature predicted by the four models including ours are shown in Fig. 7. We see from this figure that all results are almost similar. For example, the temperatures at $r=1 \mathrm{AU}$ are $120 \mathrm{~K}, 150 \mathrm{~K}$, and $160 \mathrm{~K}$, respectively, for K70, CG97, and our model. The small difference comes from the difference in $f$; noting that $f \ll 1$ and using Eqs. (9) and (26), we find $f=(q-1) z_{\mathrm{s}} / r$. Furthermore, the temperature $T$ is proportional to $f^{1 / 4}$ in the outer region where $r \geq 1 \mathrm{AU}$. By the use of Eq. (30), the results shown in Fig. 7 can be reconfirmed reasonably.

\section{Validity Check of the Adopted Assumptions}

In our present study, we have introduced some simplifications to avoid a mathematical complexity. Among these simplifications, the following two assumptions are of importance.

1) The surface, which is determined by a balance between the normal component of the wind pressure and the hydrostatic pressure, is assumed to coincide with the blackbody surface to absorb photons from the central star.

2) The wind velocity is assumed to be constant spatially over the range of the whole disk, namely from $0.03 \mathrm{AU}$ to $50 \mathrm{AU}$.

First, we consider assumption (1). In order to see the validity of assumption (1), we evaluate the optical depth, $\tau$, measured along ray paths from the central star to points inside the nebula. The depth is expressed as

$$
\tau=\kappa \int_{0}^{s} \rho(s \cos \lambda, s \sin \lambda) d s,
$$

where $\rho$ is the nebula density, $s$ is the distance from the central star along a ray path, and $\lambda$ is an angle between

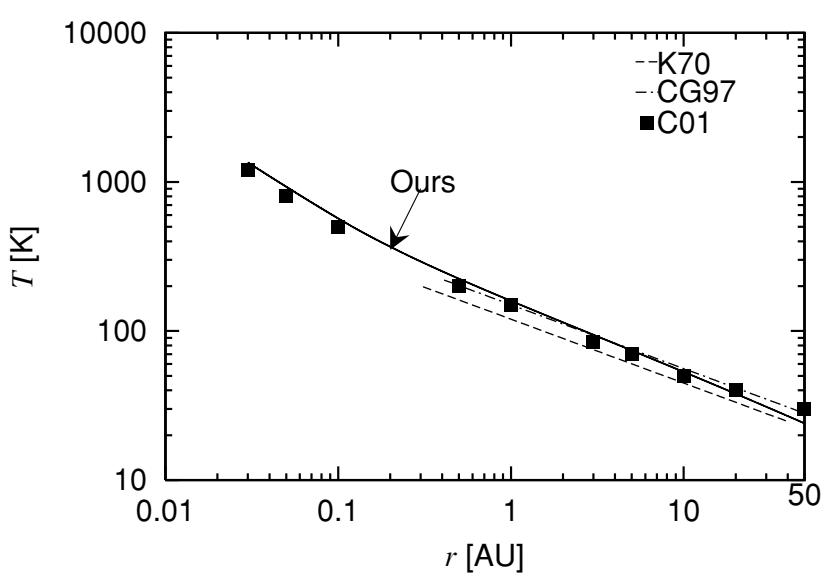

Fig. 7. Nebular temperatures predicted by various models. Solid line denotes our result for the case of $t_{*} / \beta=1 \times 10^{8} \mathrm{yrs}$. Dashed line, dotted line, and squares are temperature profiles of models of K70, CG97, and $\mathrm{C} 01$, respectively. To compare the models with the same condition, the temperature of our model and that of K70 are recalculated by using the stellar parameters, $M_{*}=0.5 \mathrm{M}_{\odot}, R_{*}=2.5 \mathrm{R}_{\odot}$, and $T_{*}=4000 \mathrm{~K}$, after CG97.

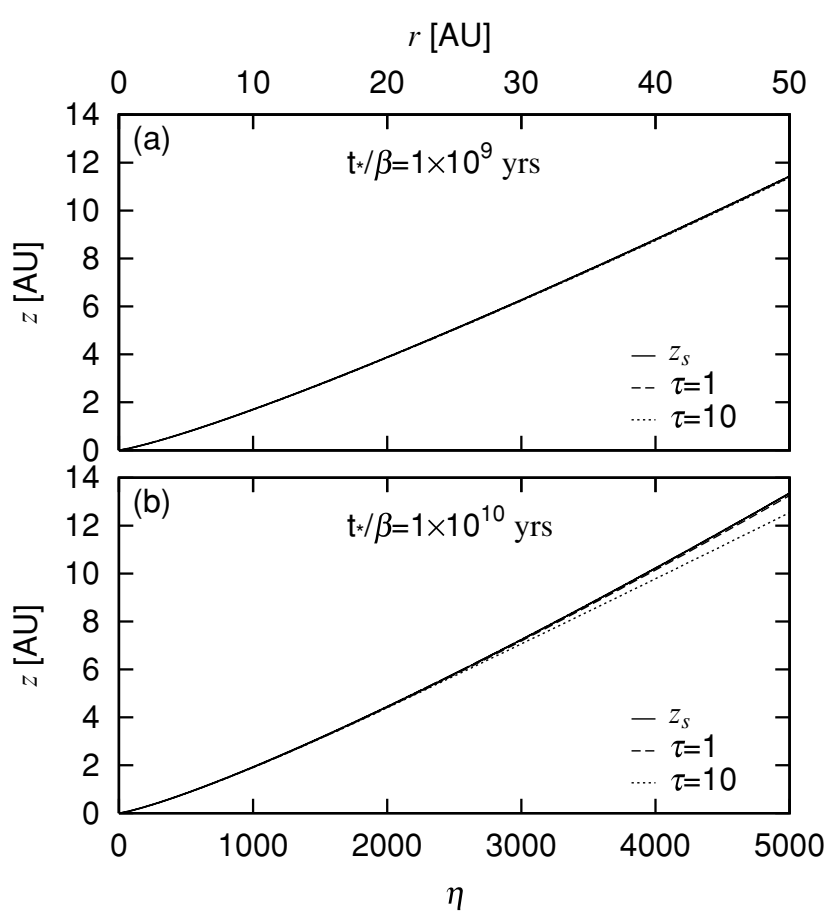

Fig. 8. Comparison between the dynamical surface and the optical surfaces of the nebula. Solid line denoted the height of the dynamical surface. Dashed and dotted lines correspond to the surface where optical depth $(\tau)$ to the stellar radiation is equal to 1 and 10 , respectively.

the ray path and the midplane of the nebula. As for the opacity $\kappa$, we use the opacity model proposed by Miyake and Nakagawa (1993), i.e., $\kappa=10 \mathrm{~cm}^{2} \mathrm{~g}^{-1}$ for radiation with a $1 \mu \mathrm{m}$ wave length. The results are shown in Fig. 8. We show the nebula height $z_{\mathrm{s}}$ (solid line) and lines of $\tau=1$ (dashed line) and $\tau=10$ (dotted line) that are evaluated from Eq. (31) for the cases of $t_{*} / \beta=1 \times 10^{9} \mathrm{yrs}$ and $1 \times 10^{10}$ yrs.

As seen from Fig. 8(a), the line of the nebular surface and the lines corresponding to $\tau=1$ and $\tau=10$ fit each other 


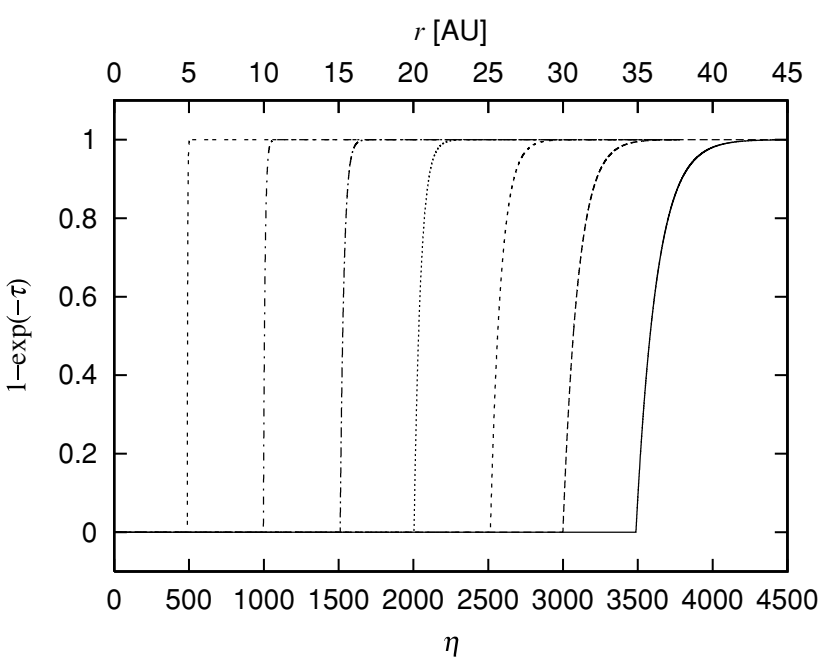

Fig. 9. Fraction of absorbed energy along the seven ray paths in the nebula (see text). We use the nebular density obtained for the case of $t_{*} / \beta=1 \times 10^{12} \mathrm{yrs}$.

completely for the case of $t_{*} / \beta=1 \times 10^{9} \mathrm{yrs}$. For the case of $t_{*} / \beta=1 \times 10^{10} \mathrm{yrs}$, the line of $\tau=1$ coincides with that of the surface (see in Fig. 8(b)), although the line of $\tau=10$ is separated slightly from the surface in the outer region $(r \geq 30 \mathrm{AU})$. From these results, we find that assumption 1 ) is valid when the wind parameter $t_{*} / \beta$ is smaller than $1 \times 10^{10} \mathrm{yrs}$.

However, as an increase in $t_{*} / \beta$ (i.e., as a decrease in the wind strength) and also as an increase in the distance from the central star, the validity of the assumption becomes doubtful because the nebula density near the surface dilutes under the weak exposure of the wind. So, we have to study in the radiative absorption for the case of $t_{*} / \beta=1 \times 10^{12} \mathrm{yrs}$ in detail. First we calculate the optical depth $\tau$ along seven different ray paths which connect the central star with points on the surface of the nebula at $r=5,10,15,20,25,30$, and 35 AU. Next, we evaluate the fraction of radiative energy absorbed by the nebular gas, $1-\exp (-\tau)$, along each ray path. The result is shown in Fig. 9. From this figure, we find that the fraction of absorbed energy increases very steeply after each radial path intersects the surface of the nebula. This is particularly evident on the ray paths which intersect the nebula surface at $5 \mathrm{AU}$ and $10 \mathrm{AU}$-all radiative energy from the central star is absorbed within a very thin region below the nebular surface. On the other hand, in the case of the ray path intersecting at $25 \mathrm{AU}$, the fraction reaches unity when the radial position takes $28 \mathrm{AU}$ or so. Even in this case, however, we can say that the black body assumption on the nebular surface is almost valid because $80 \%$ of radiative energy is absorbed within a thin layer just below the surface. Thus, we can conclude that the assumption (1) is valid almost all of the cases considered in our present study and, hence, that the nebular temperature shown in Fig. 4(a) does not need to change appreciably except the outer region $(r \geq 30 \mathrm{AU})$ of the nebula irradiated by a weak wind (with $t_{*} / \beta \geq 1 \times 10^{11} \mathrm{yrs}$ ).

Now, we go on the next problem, the check of the propriety of assumption (2) that the wind velocity is constant. Though the velocity distribution of the stellar wind is not
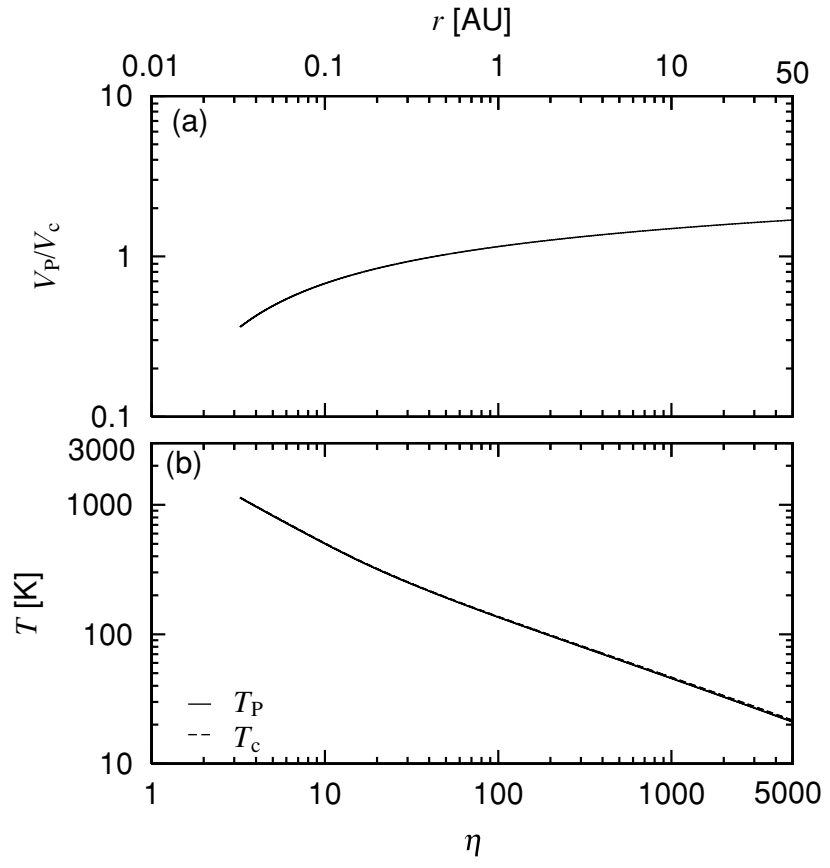

$r[\mathrm{AU}]$
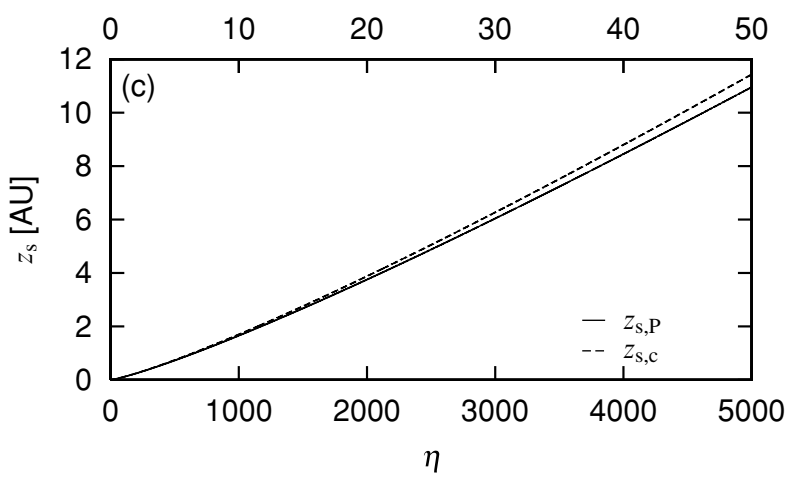

Fig. 10. The velocity, temperature and nebular height by Parker's solution. Panel (a) shows the velocity distribution, (b) the temperature distributions, and (c) the nebular height for the case of $t_{*}=1 \times 10^{9} \mathrm{yrs}$. Postscripts $\mathrm{P}$ and c denote the results calculated by Parker's solution and the constant velocity model, respectively.

detected observationally, it is natural to consider that the wind velocity varies as a function of the distance from the central star. In fact, a number of researchers proposed stellar wind models with various types of the velocity distribution (e.g., Parker, 1960; Hartmann et al., 1990; Kiguchi et al., 1998; Lamers and Cassinelli, 1999). So, we try to recalculate the temperature and the height of the nebula, as an example, using the velocity distribution proposed by Parker (1964).

The velocity distribution of Parker's solution is illustrated in Fig. 10; the wind velocity increases exponentially in the innermost region ( $r \leq 0.1 \mathrm{AU})$ with the radial distance, but in outer region $(r>0.1 \mathrm{AU})$ it grows rather gradually. As a typical example, we show the results in Figs. 10(b) and 10(c) for the case of $t_{*}=1 \times 10^{9} \mathrm{yrs}$. In these figures we overwrite our previous results for the case of $t_{*} / \beta=1 \times 10^{9} \mathrm{yrs}$. So, it is obvious that the two models give the similar results as long as we compare the models with the same wind parameter $t_{*}$. The reason is 
as follows: the vertical distribution of the nebular density changes rapidly with height $z$, because it is proportional to $\exp \left(-z^{2} / H^{2}\right)$ (see Eq. (1)) and, hence, the difference in the velocity distribution does not affect appreciably the nebular structure as long as the difference is not so serious. We also confirm that we have similar results for the other cases of $t_{*}$. Thus, the conclusion can be made that our result in the present study is plausible irrelevant to the adopted wind model as long as the mass loss rate is equal.

\section{Conclusions and Discussion}

In the this study we obtained the thermal and hydrostatical structure of the protoplanetary nebula exposed to radiation as well as the stellar wind from the central star. We have introduced the wind parameter $t_{*} / \beta$ in order to see the influence of the dynamical pressure put on the nebula by the stellar wind. This represents, strictly speaking with $\beta$ equal to unity, mass loss time. We change the parameter from $1 \times 10^{8}$ yrs to $1 \times 10^{12} \mathrm{yrs}$. After numerical calculations we arrive at the following conclusions.

1) The flaring index, $f$, of the nebula does not depend so strongly on the wind parameter $t_{*} / \beta$ and becomes large when the wind parameter is large. Owing to the suppression due to the wind pressure, the flaring index is confined within a very low level (i.e., $f<0.1$ ) in all regions.

2) The height of geometrical surface of the nebula, $z_{\mathrm{s}}$, is 2.5- to five fold as large as the scale height of the nebula. Furthermore, $z_{\mathrm{s}}$ depends very weakly on the wind parameter $t_{*} / \beta$. As the wind parameter becomes larger, the nebular height becomes slightly larger.

3) The temperature of the nebula is insensitive to the adopted wind parameter. In the inner region $(r \leq$ $0.1 \mathrm{AU})$, the nebular temperature does not depend entirely on the wind parameter $t_{*} / \beta$ because the temperature in this region is determined by irradiation from the finite size of the central star. In the outer region ( $r \geq 0.1 \mathrm{AU})$, on the other hand, the temperature is determined by the flaring index, $f$, but it still depends on the wind parameter $t_{*} / \beta$ very weakly.

4) The nebular temperature obtained in the present study is almost similar to those of the previous works (K70, CG97, and C01).

5) In the case where the wind parameter is smaller than $t_{*} / \beta=1 \times 10^{7} \mathrm{yrs}$ we have no solution to any equilibrium model of the solar nebula (see Appendix A).

It should be noted, here, that the nebular temperature obtained in our present study should be regarded as a minimum one, at least in the outer region (say, $r \geq 30 \mathrm{AU}$ ). $\mathrm{T}$ Tauri star and the surrounding disk nebula must be embedded in the extended diffuse gas and, hence, the nebula would also receive radiative energy emitted from the diffuse gas. In the outer region where the nebular temperature is very low, we cannot neglect such an additional radiative flux (Kikuchi et al., 2002).

In this study we did not carry out any analysis on the stability of the equilibrium model. It should be noted that we will meet a type of instability, the Kelvin-Helmholtz (which hereafter is called K-H) instability, near the nebular surface. It should grow more or less under the condition that large velocity discontinuities exist between the nebula and high speed wind flow. If the K-H instability is excited efficiently, the nebula may be subjected to the extensive and continuous mass loss. Furthermore, the stellar wind must change its strength with time (the wind parameter, $t_{*} / \beta$, is assumed to be constant temporarily in our present study). The time variation of the wind strength might cause other instabilities than K-H instability. These are the further problems to be solved.

We found that we have no solution describing the nebula when the stellar wind is so strong (see conclusion (5)). Although the parameter that brings non-existence of stational solution corresponds to extremely large mass loss rates, such as $10^{-7} M_{\odot}$ per year, we are greatly interested in the mathematical behavior of the basic equations. After this study we will adopt some additional processes to the basic equations, for example, to the sedimentation and rise of dusts (Ishitsu and Sekiya, 2003). Before carrying out studies based on exact models, we have better to seize the essence of the matter brought by a simple basic equations. We as yet cannot understand the physical meaning of why we have no equilibrium nebula exposed to the strong wind. As seen from the discussion in the Appendices, the condition for the non-existence of a suitable solution depends on the physical properties of the central star and the surrounding nebula, such as the stellar mass, the photospheric temperature, and the surface density of the nebula. We will investigate in detail these problems in the next paper.

\section{Appendix A.}

In this Appendix, we will study in detail the behavior of the solutions to Eq. (17) near the critical point $\left(\eta_{\mathrm{c}}, f_{\mathrm{c}}\right)$, where both $\mathcal{L}$ and $\mathcal{R}$ in Eq. (17) are equal to zero. Noted that $f_{\mathrm{c}}$ and $\eta_{\mathrm{c}}$ depend on the adopted value of the wind parameter $t_{*} / \beta$, as illustrated in Fig. 11 .

Near the critical point $\left(\eta_{\mathrm{c}}, f_{\mathrm{c}}\right), \mathcal{L}$ and $\mathcal{R}$ can be written, respectively, as

$$
\mathcal{L}(\eta, f)=\mathcal{L}_{\eta}\left(\ln \eta-\ln \eta_{\mathrm{c}}\right)+\mathcal{L}_{f}\left(\ln f-\ln f_{\mathrm{c}}\right)
$$

and

$$
\mathcal{R}(\eta, f)=\mathcal{R}_{\eta}\left(\ln \eta-\ln \eta_{\mathrm{c}}\right)+\mathcal{R}_{f}\left(\ln f-\ln f_{\mathrm{c}}\right) .
$$

In the above, $\mathcal{L}_{f}, \mathcal{L}_{\eta}, \mathcal{R}_{f}$, and $\mathcal{R}_{\eta}$ are the partial derivatives of $\mathcal{L}$ and $\mathcal{R}$ at the critical point; for example, $\mathcal{L}_{f}=$ $(\partial \mathcal{L} / \partial \ln f)_{\mathrm{c}}$. These are given by

$$
\begin{gathered}
\mathcal{L}_{\eta}=-2 \zeta_{\mathrm{c}}^{2}-\left(2 \gamma-\frac{31}{4}\right) \zeta_{\mathrm{c}}-1 \\
\mathcal{L}_{f}=-2 \zeta_{\mathrm{c}}^{2}+10 \zeta_{\mathrm{c}}-1 \\
\mathcal{R}_{\eta}=\frac{1}{64 \zeta_{\mathrm{c}}\left(1-\zeta_{\mathrm{c}}\right)}\left\{-128 \zeta_{\mathrm{c}}^{4}-32(8 \gamma-15) \zeta_{\mathrm{c}}^{3}\right. \\
-2\left(32 \gamma^{2}-48 \gamma+257\right) \zeta_{\mathrm{c}}^{2}+16(\gamma+5) \zeta_{\mathrm{c}} \\
+1\}
\end{gathered}
$$

and

$$
\mathcal{R}_{f}=2 \zeta_{\mathrm{c}}^{2}+\left(2 \gamma-\frac{31}{4}\right) \zeta_{\mathrm{c}}-\gamma-\frac{1}{8 \zeta_{\mathrm{c}}}
$$




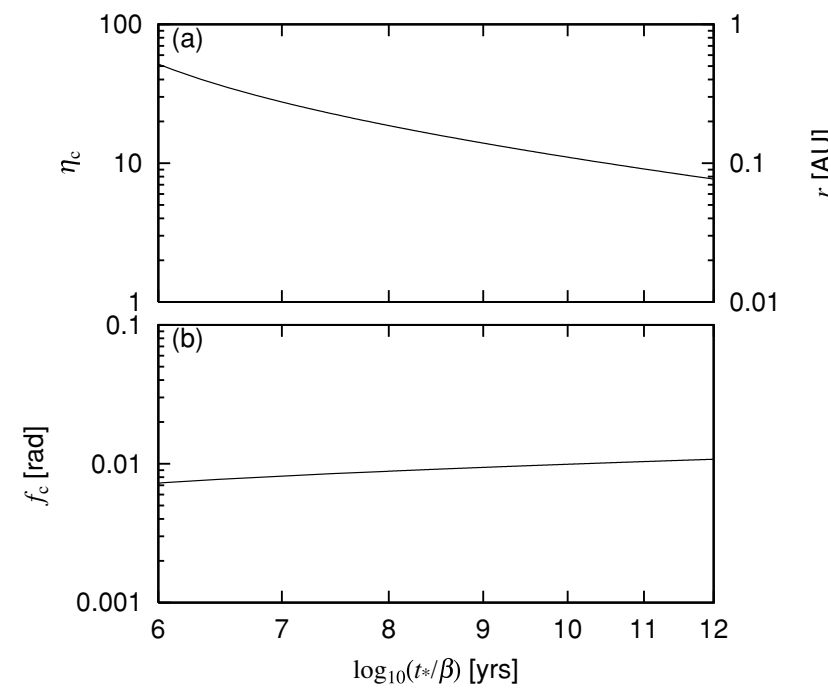

Fig. 11. Position of the critical point, $\eta_{\mathrm{c}}$ and $f_{\mathrm{c}}$, as a function of the wind parameter $t_{*} / \beta$.

where $\zeta_{\mathrm{c}}$ is a new symbol defined as

$$
\zeta_{\mathrm{c}}=\frac{1}{8}\left(\frac{3 \pi \eta_{\mathrm{c}} f_{\mathrm{c}}}{3 \pi \eta_{\mathrm{c}} f_{\mathrm{c}}+4}\right)
$$

Here we introduce a new variable, $\theta$, which is useful to the analysis of solutions to Eq. (17) near the critical point, defined by

$$
\tan \theta \equiv \frac{\ln f-\ln f_{\mathrm{c}}}{\ln \eta-\ln \eta_{\mathrm{c}}}
$$

Using $\theta$, we can rewrite Eq. (17) in the vicinity of the critical point as

$$
\frac{d \ln f}{d \ln \eta}=\frac{\mathcal{R}_{\eta}+\mathcal{R}_{f} \tan \theta}{\mathcal{L}_{\eta}+\mathcal{L}_{f} \tan \theta} .
$$

As seen in Section 3, we have two peculiar angles, $\theta_{0}$ and $\theta_{\infty}$ which are given, respectively, by

$$
\tan \theta_{0}=-\mathcal{R}_{\eta} / \mathcal{R}_{f} \quad \text { and } \quad \tan \theta_{\infty}=-\mathcal{L}_{\eta} / \mathcal{L}_{f}
$$

Note that when $\theta$ becomes equal to $\theta_{\infty}$ (or $\theta_{0}$ ), the derivative $d \ln f / d \ln \eta$ should be equal to infinity (or zero). Eliminating $\ln f$ from Eqs. (A.8) and (A.9), we have

$$
\begin{aligned}
& \left(\ln \eta-\ln \eta_{\mathrm{c}}\right) \frac{d}{d \ln \eta} \tan \theta \\
& \quad=\frac{-\mathcal{L}_{f}(\tan \theta)^{2}+\left(\mathcal{R}_{f}-\mathcal{L}_{\eta}\right) \tan \theta+\mathcal{R}_{\eta}}{\mathcal{L}_{\eta}+\mathcal{L}_{f} \tan \theta} .
\end{aligned}
$$

As is well known, a solution to Eq. (A.11) changes a functional form depending on the sign of $\mathcal{D}$, which is defined as

$$
\mathcal{D}=\left(\mathcal{L}_{\eta}+\mathcal{R}_{f}\right)^{2}+4\left(\mathcal{L}_{f} \mathcal{R}_{\eta}-\mathcal{L}_{\eta} \mathcal{R}_{f}\right) .
$$

First, we consider the case where $\mathcal{D}>0$. In this case, the solution to Eq. (A.11) can be readily found as

$$
\left|\ln \eta-\ln \eta_{\mathrm{c}}\right|^{2} \frac{\left|\tan \theta-\tan \theta_{2}\right|^{1-k}}{\left|\tan \theta-\tan \theta_{1}\right|^{-1-k}}=C,
$$

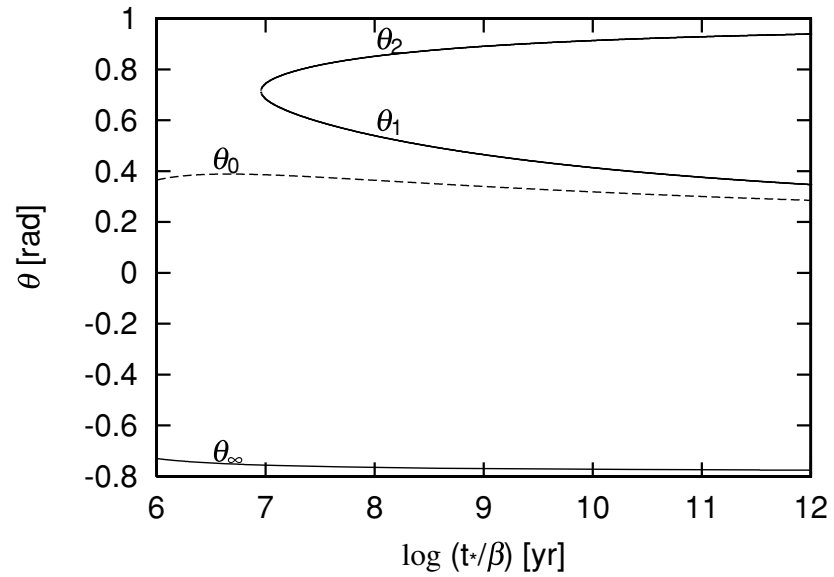

Fig. 12. Four characteristic angles $\theta_{0}, \theta_{\infty}, \theta_{1}$ and $\theta_{2}$ as functions of $t_{*} / \beta$.

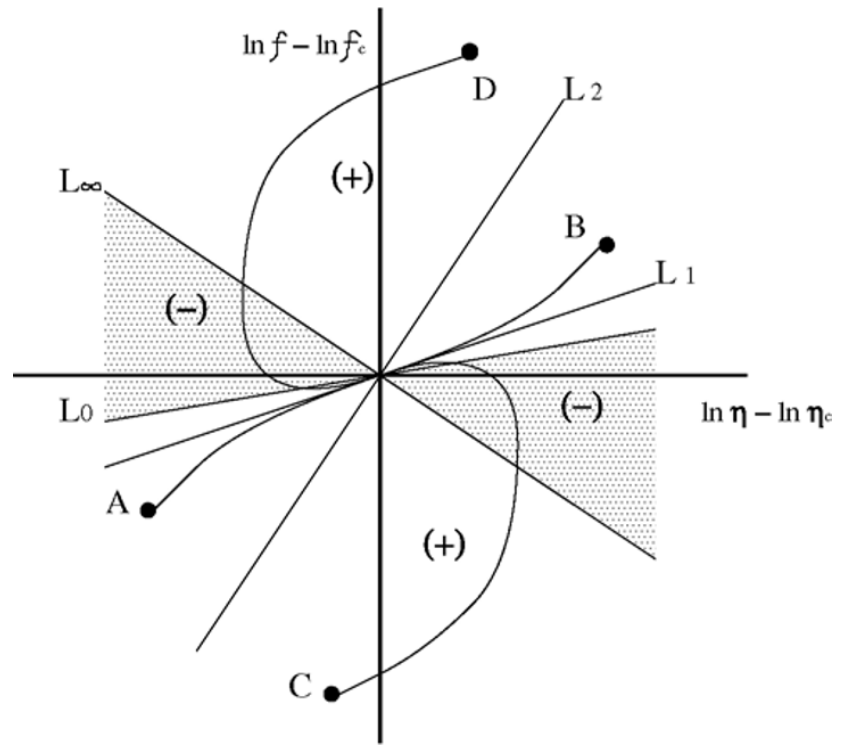

Fig. 13. Typical two solution curves near the critical point for the case of $\mathcal{D}>0$. Line $L_{\infty}$ and $L_{0}$ denote the null- $\mathcal{L}$ (that is, $d \ln f / d \ln \eta=\infty$ ) and the null- $\mathcal{R}$ (that is, $d \ln f / d \ln \eta=0$ ), namely the lines with the angle $\theta_{\infty}$ and $\theta_{0}$, respectively. Line $L_{1}$ and $L_{2}$ show the lines with the angle $\theta_{1}$ and $\theta_{2}$, respectively. In the regions denoted by (+) and (-), $d \ln f / d \ln \eta$ is positive and negative, respectively.

where $C$ is a positive constant. Furthermore, $\tan \theta_{1}$ and $\tan \theta_{2}\left(\tan \theta_{2}>\tan \theta_{1}\right)$ are given by

$$
\tan \theta_{1,2}=\frac{\mathcal{R}_{f}-\mathcal{L}_{\eta} \pm \sqrt{\mathcal{D}}}{2 \mathcal{L}_{f}}
$$

and

$$
k=\frac{\mathcal{L}_{\eta}+\mathcal{R}_{f}}{\sqrt{\mathcal{D}}} .
$$

Noting that $\mathcal{L}_{\eta}, \mathcal{L}_{f}, \mathcal{R}_{f}<0$ and $\mathcal{R}_{\eta}>0$, we readily see that $k$ is smaller than -1 . In Fig. 12 we present the characteristic four angles $\theta_{\infty}, \theta_{0}, \theta_{1}$ and $\theta_{2}$ as functions of $t_{*} / \beta$. Note that we have no real values of $\theta_{1}$ and $\theta_{2}$ when $t_{*} / \beta<1 \times 10^{7} \mathrm{yrs}$ (as long as we adopt the minimum-mass nebula model (see Eq. (1)) because $\mathcal{D}$, given by Eq. (A.12), becomes negative. As seen from this figure, $\theta_{0}$ is positive and $\theta_{\infty}$ is negative in all cases, and, $\theta_{2}$ and $\theta_{1}$ are both larger than $\theta_{0}$. 
In the limit of $\left|\ln \eta-\ln \eta_{\mathrm{c}}\right| \ll 1$, we can find an approximate, but, explicit form of Eq. (A.13). We first consider a solution passing through, for example, point $\mathrm{B}$ in Fig. 13, at which $\tan \theta_{2}>\tan \theta_{B}>\tan \theta_{0}$. In this case, we have for a limiting form of Eq. (A.13)

$$
\left|\tan \theta-\tan \theta_{1}\right|=C^{\prime}\left|\ln \eta-\ln \eta_{\mathrm{c}}\right|^{-\frac{2}{k+1}},
$$

where $C^{\prime}=\left\{C\left(\tan \theta_{2}-\tan \theta_{1}\right)^{k-1}\right\}^{\frac{1}{k+1}}$. From this equation, we can readily see that such a solution passes through the critical point with a derivative, $(d \ln f / d \ln \eta)_{\mathrm{c}}=\tan \theta_{1}$, and escapes continuously to the region of negative $\ln \eta-$ $\ln \eta_{\mathrm{c}}$. An alternative solution which passes through, say point $\mathrm{D}$ in Fig. 13 at which $\tan \theta_{\mathrm{D}}>\tan \theta_{2}$, behaves, in a region of $\left|\ln \eta-\ln \eta_{\mathrm{c}}\right| \ll 1$, as

$$
\begin{aligned}
\tan \theta\left(\ln \eta-\ln \eta_{\mathrm{c}}\right)= & \ln f-\ln f_{\mathrm{c}} \\
= & \frac{1}{2}\left\{(1-k) \tan \theta_{2}+(1+k) \tan \theta_{1}\right\} \\
& \times\left(\ln \eta-\ln \eta_{\mathrm{c}}\right)+\sqrt{C} .
\end{aligned}
$$

Solution (A.17) cuts the $\ln f$-axis with a finite $f$.

From the above results, we can find that there are two different types of solutions to Eq. (A.11) when $\mathcal{D}>0$, as illustrated in Fig. 13. In this figure, we overwrite the four characteristic lines, $L_{\infty}, L_{0}, L_{1}$, and $L_{2}$, with slopes of $\tan \theta_{\infty}, \tan \theta_{0}, \tan \theta_{1}$, and $\tan \theta_{2}$, respectively. As discussed in Section 3 , the solution curve has a positive gradient in the shaded regions bounded by $L_{\infty}$ and $L_{0}$, whereas it has a negative gradient in the other two regions.

A typical example of the first type solution is presented in Fig. 13 as curve A to B. This solution passes through the critical point along asymptoticly line $L_{1}$ with angle $\theta_{1}$. It should be noted that a solution of this type never crosses line $L_{2}$ because of Eq. (A.13). This means that such a solution is confined within regions bounded by lines $L_{1}$ and $L_{2}$ where $d \ln f / d \ln \eta$ is always positive. So the solution is a monotonically increasing function of $\ln \eta$.

Another type of solution is displayed schematically in Fig. 13 as curve $C$ to $D$. Since the derivative is positive at point $\mathrm{C}$, the solution curve goes upward in the $\ln \eta$ - $\ln f$ plane as $\eta$ increases and crosses line $L_{\infty}$ vertically. Thereafter, the solution curve enters the region where the derivative is negative and continues to increase in $f$ but decrease in $\eta$. After the solution line crosses line $L_{0}$ horizontally, it runs toward the critical point according to Eq. (A.16). As mentioned before, Eq. (A.9) has a point symmetry to the critical point. We can draw the solution curve after it passes through the critical point by rotating the solution curve from $\mathrm{C}$ around the critical point by $180^{\circ}$. It is worthwhile saying that the above solution is a multivalue function of $\eta$, at least in a region around the critical point, and that we cannot adopt it as a suitable solution because such a solution can never describe the nebula model.

We next consider another case where $\mathcal{D}$, given by Eq. (A.12), is negative. In this case, the solution to Eq. (A.11) is given by the parametric equations:

$$
\ln \eta-\ln \eta_{\mathrm{c}}=C \exp (-k \varphi) \cos \varphi
$$

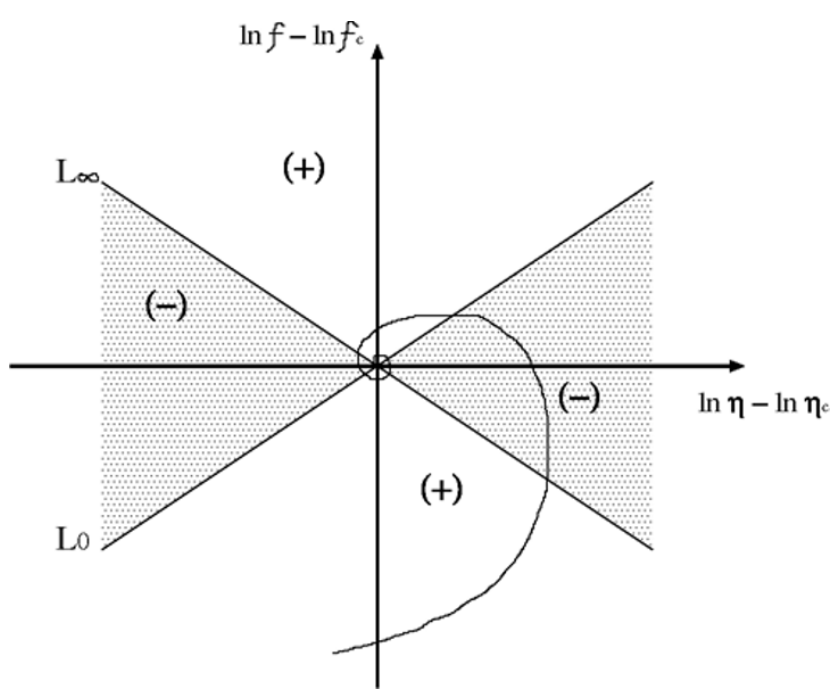

Fig. 14. Schematic picture of the solution near the critical point for the case of $\mathcal{D}<0$ (see also the caption of Fig. 13).

and

$$
\begin{aligned}
\ln f-\ln f_{\mathrm{c}}= & \frac{C}{2 \mathcal{L}_{f} k} \exp (-k \varphi)\left\{\left(\mathcal{L}_{\eta}+\mathcal{R}_{f}\right) \sin \varphi\right. \\
& \left.-k\left(\mathcal{L}_{\eta}-\mathcal{R}_{f}\right) \cos \varphi\right\}
\end{aligned}
$$

where $C$ is an integral constant and $d \varphi / d \theta$ is a negative for $0<\theta<\infty$. The schematical view of the solution given by Eqs. (A.18) and (A.19) is shown in Fig. 14. The solution curve spirals around the critical point getting closer to the critical point. Owing to the exponential factor, when the parameter $\varphi$ decreases, the solution curve terminates rapidly at the critical point. By the same reason as mentioned earlier, we cannot accept any of the solutions for the case of $\mathcal{D}<0$ as those describing the physical model of the solar nebula.

From our analysis on the behavior of solutions to Eq. (A.9) near the critical point, we reach the following important conclusions.

1) In order to look for a solution describing the nebula model which extends from the inside of the critical point to the outside, we should choose a solution from solutions which passes through the critical point and which runs in regions bounded by the two lines $L_{1}$ and $L_{2}$.

2) When the wind parameter is small (i.e., when the wind is strong), we have no solution describing the nebula model. In the present study in which the surface density of the nebula distributes according to the minimum solar nebula model (see Eq. (1)), we have no solution when $t_{*} / \beta<1 \times 10^{7}$ yrs.

\section{Appendix B.}

We have to impose an appropriate boundary condition on $f$ at outer (or inner) boundary of the nebula to solve Eq. (17), though it is difficult to do it. So we impose boundary conditions on $f$. We then choose an appropriate solution imposing two conditions. In Fig. 15(a) we present eight solution curves with different outer boundary conditions. 


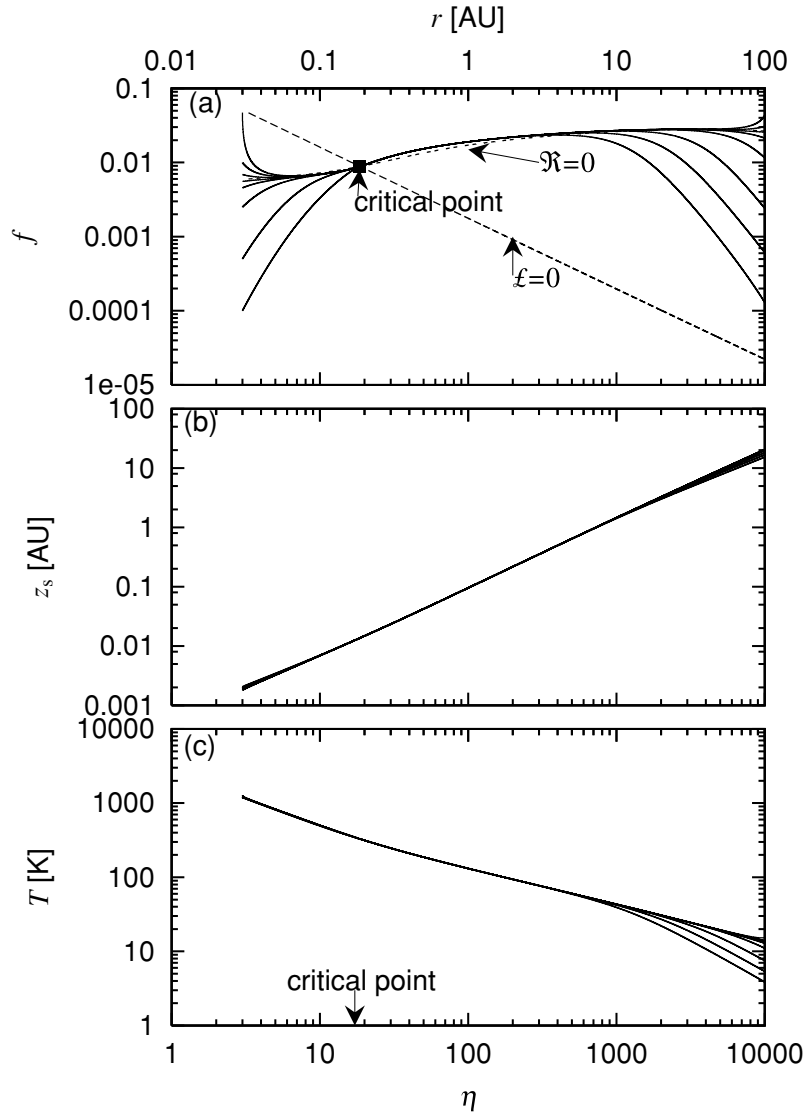

Fig. 15. Solutions of the Eq. (17) for this case $t_{*} / \beta=1 \times 10^{8}$ yrs. Plots are for (a) flaring index, $f(\mathrm{~b})$ height of the surface, $z_{\mathrm{s}}$ and (c) temperature distribution with outer and inner different boundary conditions.

As easily seen from this figure, solution curves extending from outer boundary converge to one curve smoothly as the coordinate $r$ decrease. The converged curve approaches the critical point as an asymptote line with angle $\theta_{1}$ explained in Appendix A. In Fig. 15(b) we present eight solutions with different inner boundary conditions. As in the case of integration from the outer boundary, the solution curves converge to one curve smoothly before they reach the critical point.

The value of $f$ depends on outer and inner boundary conditions near each boundary position, though temperature distribution does not. In Fig. 15(c) we show nebula temperature given from the 16 solutions. In the inner region, $f$ is much smaller than the second term in right hand side of Eq. (16). So temperature in this inner region does not depend on $f$ (see Fig. 3). As the radius $r$ increases, the temperature gradually depends on $f$, then at the outer boundary the temperature for eight different conditions differs greatly. These differences correspond to outer boundary conditions of $f$ directly. From Fig. 15(c) we consider that as long as we need the temperature profile of the nebula only at the inner part, namely more than about $10 \mathrm{AU}$ inner, we can use the converged curve independently from outer boundary conditions. So we concentrated on the converged curve. For practical treatment, we impose the condition, $\mathcal{R}=0$, on the outer boundary.

\section{References}

Adams, F. C., C. J. Lada, and F. H. Shu, Spectral evolution of young stellar object, Astrophys. J., 312, 788-806, 1987.

Adams, F. C., C. J. Lada, and F. H. Shu, The disk of T Tauri stars with flat infrared spectra, Astrophys.J., 326, 865-883, 1988.

Basri, G. and C. Bertout, Accretion disks around T Tauri Stars. II. Balmer emission, Astrophys. J., 341, 340-358, 1989.

Beckwith, S. V. W., A. I. Sargent, R. S. Chini, and R. Gusten, A survey for circumstellar disks around young stellar objects, Astron. J., 99, 924945, 1990.

Bertout, C., T Tauri Stars: Wild as dust, Annu. Rev.Astron. Astrophys., 27, 351-395, 1989.

Boss, A. P., Evolution of the solar nebula. II. Protoplanetary disks undergoing mass accretion, Astrophys. J., 469, 906-920, 1996.

Calvet, N., Properties of the wind of T Tauri stars, IAUS, 182, 417-432, 1997

Carr, J. S., Near-infrared CO emission in young stellar objects, Astrophys. $J ., 345,522-535,1989$.

Chiang, E. I. and P. Goldreich, Spectral energy distributions of T Tauli stars with passive circumstellar disks, Astrophys. J., 490, 368-376, 1997.

Chiang, E. I., M. K. Joung, M. J. Creech-Eakman, C. Qi, J. E. Kessler, G. A. Blake, and E. F. van Dishoeck, Spectral energy distributions of passive T Tauri and Herbig Ae disks: grain mineralogy, parameter dependences, and comparison with infrared space observatory LWS observations, Astrophys. J., 547, 1077-1089, 2001.

Greene T. P. and M. Meyer, An infrared spectroscopic survey of the $\rho$ Ophiuchi young stellar cluster: Masses and ages from the H-R diagram, Astrophys. J., 450, 233-244, 1995.

Hartigan, P., S. Edwards, and L. Ghandour, Disk Accretion and loss from young stars, Astrophys. J., 452, 736-768, 1995.

Hartmann, L., Mass loss from solar-type stars, Solar Phys., 100, 587-597, 1985.

Hartmann, L., N. Calvet, E. Avrett, and R. Loeser, Winds from T Tauri stars. I. Spherically symmetric models, Astrophys. J., 349, 168-189, 1990.

Hartmann, L., N. Calvet, E. Avrett, and P. D'Alessio, Accretion and the evolution of T Tauri Disks, Astrophys. J., 495, 385-400, 1998.

Hayashi, C., K. Nakazawa, and Y. Nakagawa, Formation of the Solar System, in Protostars and Planets II, Edited by Black, D. C. and M. S. Matthews, 1100-1153 pp, Tucson, Univ. Arizona Press, 1985.

Ishitsu, N. and M. Sekiya, The effects of the tidal force on shear instabilities in the dust layer of the solar nebula, Icarus, 165, 181-194, 2003.

Kenyon, S. J., I. Yi, and L. Hartmann, A magnetic accretion disk model for the infrared excesses of T Tauri stars, Astrophys. J., 462, 439-455, 1996.

Kiguchi, M., S. Narita, and C. Hayashi, Wind from T Tauri stars, PASJ, 50, 587-595, 1998.

Kikuchi, N., T. Nakamoto, and K. Ogochi, Disk-halo model for flatspectrum T Tauri stars, PASJ, 54, 589-597, 2002.

Kuhi, L., Mass loss from T Tauri stars, Astrophys. J., 140, 1409-1432, 1964

Kusaka, T., T. Nakano, and C. Hayashi, Growth of solid particles in the primordial solar nebula, Progr. Theor. Phys., 44, 1580-1595, 1970.

Lamers, H. J. G. L. M. and J. P. Cassinelli, Introduction to Stellar Wind, Camprige Univ. Press, Cambrige, 1999.

Lin, D. N. C. and J. C. B. Papaloizou, On the Dynamical Origin of the Solar System, in Protostars and Planets II, Edited by Black, D. C. and Matthews, M. S., 981-1072, Univ. Arizona Press, Tucson, 1985.

Lin, D. N. C. and J. C. B. Papaloizou, Theory of accretion disks II: Application to observed systems, Annu. Astron. Astrophys., 34, 703-747, 1996.

Lynden-Bell, D. and E. Pringle, The evolution of viscous discs and the origin of the nebular variables, MNRAS, 168, 603-637, 1974.

McCaughrean, M. J. and C. R. O'Dell, Direct imaging of circumstellar disks in the Orion nebula, Astron. J., 111, 1977-1987, 1996.

Miyake, K. and Y. Nakagawa, Effects of particle size distribution on opacity curves of protoplanetary disks around T Tauri stars, Icarus, 106, 20 41, 1993.

Papaloizou, J. C. B. and D. N. C. Lin, Theory of accretion disks I: Angular momentum transport processes, Ann. Astron. Astrophys., 33, 505-540, 1995.

Parker, E. N., The hydrodynamic Theory of Solar Corpuscular Radiation and Stellar Winds, Astrophys. J., 132, 821-866, 1960.

Parker, E. N., Dynamical properties of stellar coronas and stellar winds.I. Integration of the momentum equation, Astrophys. J., 139, 72-92, 1964. 
Rydgren, A. E. and D. S. Zak, On the spectral from of the infrared excess component in T Tauri systems, PASP, 99, 141-145, 1987.

Safronov, V.S., Evolution of the Protoplanetary Cloud and Formation of the Earth and Planets, Nauka, Moscow., 1969.; Transl. NASA TTF$677,1972$.

Strom, K. M., S. E. Strom, S. Edwards, S. Cabrit, and M. F. Skruskie, Circumstellar material associated with solar-type pre-main-sequence stars: A possible on the timescale for planet building, Astron. J., 97, 14511470, 1989.
Walter, F. M., A. Brown, R. D. Mathieu, P. C. Myers, and F. J. Vrba, X-ray sources in regions of star formation. III. Naked T Tauri Stars associated with the Taurus-Auriga Complex, Astron. J., 96, 297-325, 1988.

Wood, B. E., H.-R. Muller, G. P. Zank, and J. L. Linsky, Measured massloss rates of solar-like stars as a function of age and activity, Astrophys. J., 574, 412-425,2002.

Y. S. Yun (e-mail: yun@geo.titech.ac.jp), H. Emori, and K. Nakazawa 